\title{
CLINICAL PRESENTATION OF COVID19 IN DEMENTIA PATIENTS
}

\author{
A. BIANCHETTI ${ }^{1,4}$, R. ROZZINII ${ }^{2,4}$, F. GUERINI ${ }^{1,4}$, S. BOFFELLI ${ }^{2,4}$, P. RANIERI ${ }^{1,4}$, G. MINELLI $^{1,4}$, \\ L. BIANCHETTI ${ }^{3,4}$, M. TRABUCCHI ${ }^{4}$
}

\begin{abstract}
1. Medicine and Rehabilitation Department, Istituto Clinico S.Anna Hospital, Brescia, Italy; 2. Geriatric Department, Fondazione Poliambulanza Istituto Ospedaliero Hospital; Brescia, Italy; 3. Geriatric Reahabilitation Unit, Anni Azzurri, Rezzato, Brescia, Italy; 4. Italian Association of Psychogeriatrics, Italy; All the authors contributed equally to the drafting of this

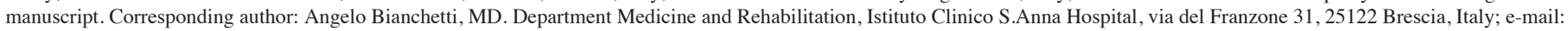
angelo.bianchetti@grupposandonato.it, https://orcid.org/0000-0002-2914-0627, phone: +390303197409 - fax: +390303198687
\end{abstract}

\begin{abstract}
Objective: No studies analyzing the role of dementia as a risk factor for mortality in patients affected by COVID-19. We assessed the prevalence, clinical presentation and outcomes of dementia among subjects hospitalized for COVID19 infection. Design: Retrospective study. Setting: COVID wards in Acute Hospital in Brescia province, Northern Italy. Participants: We used data from 627 subjects admitted to Acute Medical wards with COVID 19 pneumonia. Measurements: Clinical records of each patients admitted to the hospital with a diagnosis of COVID19 infection were retrospectively analyzed. Diagnosis of dementia, modalities of onset of the COVID-19 infection, symptoms of presentation at the hospital and outcomes were recorded. Results: Dementia was diagnosed in 82 patients $(13.1 \%)$. The mortality rate was $62.2 \%(51 / 82)$ among patients affected by dementia compared to $26.2 \%(143 / 545)$ in subjects without dementia $(\mathrm{p}<0.001$, Chi-Squared test). In a logistic regression model age, and the diagnosis of dementia resulted independently associated with a higher mortality, and patients diagnosed with dementia presented an OR of 1.84 (95\% CI: 1.09-3.13, p<0.05). Among patients diagnosed with dementia the most frequent symptoms of onset were delirium, especially in the hypoactive form, and worsening of the functional status. Conclusion: The diagnosis of dementia, especially in the most advanced stages, represents an important risk factor for mortality in COVID-19 patients. The clinical presentation of COVID-19 in subjects with dementia is atypical, reducing early recognition of symptoms and hospitalization.
\end{abstract}

Key words: COVID19 infection, dementia, mortality risk.

\section{Introduction}

In Italy, SARS-CoV-2 outbreak was catastrophic with 135,586 confirmed cases and 17,127 deaths at April, 8th (1). In clinical series of patients who died of COVID-19 comorbidities (especially hypertension, cardiac ischemic disease, diabetes and obesity) were identified as significant risk factors for mortality, while dementia was described as a comorbid condition in only $6.8 \%$ of COVID-19 patients (2).

Although dementia is known to be an important mortality risk factor among older people, so far there are no studies analyzing the role of dementia as a risk factor for mortality in patients affected by COVID-19 $(3,4)$.

In the Province of Brescia, an administrative district in eastern Lombardy home to 1.2 million people, between February 22nd and April 8th, 9,900 cases of Covid-19 have been diagnosed and 1,800 deaths have been reported. About $53 \%$ (2265 out of 4200) of hospital beds have been dedicated to treat patients affected by Covid-19 pneumonia. Specific units were created to cater to these patients: acute medical units, named COVID Wards, and intensive care units, with the last accounting fort the $8.5 \%$ of all the beds dedicated to COVID-19 patients.

\section{Methods and study population}

During this period, 627 patients diagnosed with COVID19 pneumonia were admitted to our hospitals. All patients admitted to COVID Wards were positive to RT-PCR for SARSCov-2 conducted on a nasopharyngeal specimen and presented respiratory failure. Each patient underwent a thorough medical evaluation and, if over 65, a geriatric multi-dimensional assessment, comprehensive of evaluation of cognitive and functional status and presence of delirium.

Dementia was diagosed according to clinical history and results of the cognitive assessment. The modalities of onset of the COVID-19 infection, the symptoms of presentation at the hospital emergency department and the outcomes were recorded.

\section{Results}

Dementia was diagnosed in 82 patients $(13.1 \%)$. The mean age of patients diagnosed with dementia was 82.6 (SD 5.3; IQR 80-86), versus 68.9 (SD 12.7; IQR 60-68) in patients not affected by dementia ( $\mathrm{p}<0.001$; Student's t test). Females were $47(57.3 \%)$ among patients with dementia and $288(52.8 \%)$ among patients not diagnosed with dementia, respectively.

The mortality rate was $62.2 \%(51 / 82)$ among patients affected by dementia compared to $26.2 \%$ (143/545) in subjects without dementia $(\mathrm{p}<0.001$, Chi-Squared test). (Table 1)

The Clinical Dementia Rating Scale (CDR) (5) was used to determine the severity of dementia: 36 patients $(43.4 \%)$ were classified in stage 1, $15(18.3 \%)$ in stage 2 and $31(37.8 \%)$ in stage 3 . The Mortality rates were, respectively, 41.7\%, 66.7\%, and $83.9 \%(\mathrm{p}<0.001$, one-way ANOVA) (table 2). 
Table 1

Characteristics of 627 patients consecutively hospitalized for COVID19 pneumonia in two Italian hospitals according to the diagnosis of dementia

\begin{tabular}{|c|c|c|c|c|}
\hline Characteristics & Sample & Dementia & no Dementia & $\mathbf{p}$ \\
\hline No, $(\%)$ & $627(100)$ & $82(13.1)$ & $545(86.9)$ & \\
\hline \multicolumn{5}{|l|}{ Sex, No (\%) } \\
\hline women & $335(53.4)$ & $47(57.3)$ & $288(52.8)$ & \\
\hline Age, mean (SD), y & 70,7 (12.9) & $82.6(5.3)$ & $68.9(12.7)$ & $<0.001 * *$ \\
\hline
\end{tabular}

* Pearson's chi-squared test; ** Student's t-test

Table 2

Characteristics of 627 patients consecutively hospitalized for COVID19 pneumonia in two Italian hospitals according to CDR classification

\begin{tabular}{|c|c|c|c|c|c|}
\hline Characteristics & CDRO & CDR1 & CDR2 & CDR3 & $\mathbf{p}$ \\
\hline No, $(\%)$ & $545(86.9)$ & $36(5.8)$ & 15. (2.4) & $31(5.0)$ & \\
\hline \multicolumn{6}{|l|}{ Sex, No $(\%)$} \\
\hline men & $257(47.2)$ & $20(55.6)$ & $5(33.3)$ & $10(32.3)$ & NS* \\
\hline women & $288(52.8)$ & $16(44.4)$ & $10(66.7)$ & $21(67.7)$ & \\
\hline Age, mean (SD), y & $68.9(12.7)$ & $82.0(5.1)$ & $83.0(7.4)$ & $83.1(4.2)$ & $<0.001 *$ \\
\hline Mortality, no (\%) & $143(26.2)$ & $15(41.7)$ & $10(66.7)$ & $26(83.9)$ & $<0.001 *$ \\
\hline
\end{tabular}

* one-way ANOVA

Table 3

Binary Logistic Regression Model for mortality by Age, Sex and Dementia

\begin{tabular}{lccc}
\hline Predictors & Odds Ratio & 95\% CI & p \\
\hline Age, 1-year increase & 1.09 & $1.07-1.12$ & $<0.001^{*}$ \\
Sex, female & 0.87 & $0,60-1.27$ & NS* \\
Dementia & 1.84 & $1.08-3.13$ & $0,024^{*}$ \\
\hline
\end{tabular}

* Wald Test for Analysis of Variance

To assess if the diagnosis of dementia was associated with a worse outcome regardless of age and sex, we built a logistic regression model. According to this model age, and the diagnosis of dementia resulted independently associated with a higher mortality. For every increased year of age, the Odds Ratio (OR) for mortality was 1.09 (95\% CI: 1.07-1.12, $\mathrm{p}<0.001)$, and patients diagnosed with dementia presented an OR of 1.84 (95\% CI: $1.09-3.13, \mathrm{p}<0.05)$. According to this model sex was not associated with a change in mortality risk. (Table 3)

As shown in table 4, among patients diagnosed with dementia the most frequent symptoms of onset were delirium (67\%, especially in the hypoactive form, 50\%) and worsening of the functional status. The classic symptoms of COVID-19 infection were less frequent: only $47 \%$ of patients had fever, $44 \%$ dyspnea and $14 \%$ cough.

\section{Conclusions}

Caring for patients with dementia during the current pandemic is a complex task, involving the management of patients in different settings. Some patients need to be treated at home, often with caregivers burdened by isolation due to lockdown measures and by limitation of home services. Other patients are cared in nursing homes, which often lack adequate and trained staffs and access to personal protective equipment. 


\section{CLINICAL PRESENTATION OF COVID19 INFECTION IN DEMENTIA PATIENTS}

Hospital patient's management has been difficult due to the scarce collaboration offered by the patient and difficulties in communication, immobility, and limited availability of trained staff members (6). There are also ethical concerns regarding hospitalization of patients with dementia due to resource constraints during the current pandemic (7).

\section{Table 4}

Symptoms at ER admission among 82 dementia patients consecutively hospitalized for COVID19 pneumonia in two Italian hospitals

\begin{tabular}{lc}
\hline Symptoms, No $(\%)$ & \\
\hline Delirium & $55(67.1)$ \\
Hypoactive & $41(50.0)$ \\
Hyperactive & $17(20.7)$ \\
Functional status worsening & $46(56.1)$ \\
Behavioral symptoms & $9(11.0)$ \\
Fever & $39(47.6)$ \\
Cough & $11(13.4)$ \\
Dyspnea & $36(43.9)$ \\
\hline
\end{tabular}

To our knowledge, the proportion of subjects with dementia among patients admitted to an acute hospital for COVID19 has never been evaluated. The prevalence of demented patient found in the present study $(13.1 \%)$ is lower than the previous estimates of the prevalence of dementia in hospital, which vary from $15 \%$ to $42 \%$ (7). According to our data, the diagnosis of dementia, especially in the most advanced stages, represents an important risk factor for mortality in COVID-19 patients. The clinical presentation of COVID-19 in subjects with dementia is atypical, reducing early recognition of symptoms and hospitalization. We suggest that the onset of hypoactive delirium and worsening functional status in people with dementia may be considered a sign of possible COVID-19 infection during this epidemic. Early recognition of COVID19 in demented people can help provide timely treatment and adequate isolation. Hospitals should develop integrated care models, create Special Care Geriatric COVID units and promote guidelines to ensure the better possible treatment for frail older persons.

Funding: No funding.

Conflicts of interest/Competing interests: The authors declare that they have no conflict of interest.

Ethics approval: This is a review study; the protocol was approved by the institutional committee.

Acknowledgement: Anita Chizzoli, MD; Marzia Cristo, MD; Silvia Comini, MD; Assunta Di Stasio, MD and Antonella Ricci, MD for the support in clinical evaluation of patients.

\section{References}

1. Word Health Organization. Coronavirus disease 2019 (COVID-19) Situation Report - 79. Available at: https://www.who.int/docs/default-source/coronaviruse/situationreports/20200408-sitrep-79-covid-19.pdf?sfvrsn=4796b143_4

2. Onder G, Rezza G, Brusaferro S. Case-Fatality Rate and Characteristics of Patients Dying in Relation to COVID-19 in Italy. JAMA. Published online March 23, 2020. doi:10.1001/jama.2020.4683

3. Morandi A, Di Santo SG, Zambon A et al, Italian Study Group on Delirium (ISGoD). Delirium, Dementia, and In-Hospital Mortality: The Results From the Italian Delirium Day 2016, A National Multicenter Study. J Gerontol A Biol Sci Med Sci;2019;74:910916.

4. D'Adamo H, Yoshikawa T, Ouslander JG. Coronavirus Disease 2019 in Geriatrics and Long-term Care: The ABCDs of COVID-19. JAGS 2020;doi: 10.1111/jgs.16445

5. Hughes CP, Berg L, Danziger WL, Coben LA, Martin RL. A new clinical scale for the staging of dementia. The British Journal of Psychiatry; 1982;140, 566-572.

6. Wang H, Li T, Barbarino P, Gauthier S, Brodaty H, Molinuevo JL, Xie H, Sun Y, Yu E, Tang Y, Weidner W, Yu X. Dementia care during COVID-19. Lancet. 2020;395(10231):1190-1191. doi: 10.1016/S0140-6736(20)30755-8. Epub 2020 Mar 30 .

7. Aprahamian I, Cesari M. Geriatric Syndromes and SARS-COV-2: More than Just Being Old [published online ahead of print,]. J Frailty Aging. 2020;1-3. doi:10.14283/ jfa.2020.17

8. Jackson TA, Gladman JR, Harwood RH et al. Challenges and opportunities in understanding dementia and delirium in the acute hospital. PLoS Med:e 2017;1002247. 